\title{
Research and Simulation on Wireless Packet Scheduling Scheme Based on High-Speed Packet Access Network
}

\author{
Hu Linna ${ }^{*}, 1,2$, Cao Ning ${ }^{1}$ and Mao Minghe ${ }^{1}$ \\ ${ }^{1}$ Computer \& Information College, Hohai University, Jiangsu 210098, China \\ ${ }^{2}$ Department of Dianguang, Nanjing University of Science \& Technology Zijin College, Jiangsu 210046, China
}

\begin{abstract}
The efficiency of high speed packet access (HSPA) systems is mainly based on Packet Scheduling Scheme which will be the key to influencing on the system performance and ensuring the service quality for the users. In this paper, we introduce a three step packet scheduling scheme to provide quality of services (QoS) guaranteed for high speed uplink packet access. Then, we proposed an improved packet scheduling algorithm based on QoS parameter for mixed service applications in order to meet the actual QoS needs of the different systems. This algorithm calculated the scheduling priority based on the comprehensive factors, including the queuing status of each user, QoS requirements and the sum of speeds on each channel. The simulation results show that the performance efficiency of HSPA systems employed proposed algorithm is superior compared to the conventional PF and M-LWDF algorithm in average packet drop and average cell throughput.
\end{abstract}

Keywords: HSPA, packet scheduling, priority, QoS.

\section{INTRODUCTION}

Nowadays, HSPA is the best communication system to provide different services with QoS guaranteed to UEs at any time, any place, and supporting full mobility in a costeffective and efficient manner. HSPA is also the best available radio-based technology when considering high download and upload speeds. The uplink is becoming increasingly important, with many UEs interested in uploading their clips onto online streaming providers and similar video-sharing sites [1,2].

In the system of HSPA, the function of radio resource management is mainly achieved by packet scheduling algorithm which will be the key to influence the system performance and ensuring the service quality for the users. Research on advanced packet scheduling algorithm is required to improve the data throughput, guarantee the fairness between users and meet the QoS requirements. Proper scheduling can make the users share the link bandwidth more fairly; enhance the perception of the users; enable the network to have a larger data service capacity; enhance the effectiveness of network construction. Therefore, it is really necessary to combine with the characteristics of the system and the diversified types of services to find out a suitable scheduling algorithm on the HSPA network platform.

The HSDPA scheduler is the key to resource management in the UTRAN downlink because it decides which user is to be scheduled in each transmission time interval (TTI) [3]. In general, the scheduling decision does not take into account only the channel conditions, but additional factors such as fairness between users, cell throughput, and QoS parameters are typically considered in the scheduling mechanism. The choice of a scheduler usually involves some trade-offs between these factors.

MAC (mediam access control) layer packet scheduling is one of the salient points of HSPA to perform resource management (i.e., bandwidth allocation between terminals), taking into account the radio channel conditions of all users. In some proposals, additional factors such as fairness between users or cell throughput or quality-of-service (QoS) parameters are also considered in the scheduling mechanism [4].

Among all the scheduling algorithms supporting realtime traffic flow, M-LWDF is applied more widely for its priority function takes the packet drop rate, head of line packet delay and the channel conditions of user equipment into account so as to provide high quality of service for users [5]. However, this algorithm has obvious defects: 1) it fails to solve the resource allocation problem between the realtime user and non-real-time user; 2) the fairness between these two users in getting access to services is not guaranteed, so that he single algorithm such as PF and MLWDF is not applicable to hybrid real-time /non real-time traffic scheduling [6].

Among the existing non-real-time scheduling algorithms, PF has been widely applied. PF arranges the priority function of each user before scheduling decision and perform scheduling to the priority services. It enables users to have long-term fairness to get access to the services and improves the throughput of user equipment. However, the real-time 
traffic flows have strict delay constraints so that $\mathrm{PF}$ algorithm is not applicable to real-time services (such as video and voice) [7].

QoS guarantees will be required by users of services such as video streaming that have requirements in terms of minimum bit rate and maximum packet delay. User satisfaction is the main factor to consider. Satisfaction criteria are different for different services, each having its own QoS requirements. Thus, packet scheduling should aim to fulfill these QoS requirements instead of just focusing on cell throughput and fairness [8]. QoS classes and parameters have already been defined by 3GPP standards [9]. However, these standards do not define the scheduler implementation details, and thus provide flexibility to implementers and Node B vendors to choose their own scheduler in order to map these QoS classes and parameters.

How to reasonably allocate resources for a variety of service with different bandwidth requirements and different QoS levels to meet their basic service needs, and how to improve the overall network throughput and spectrum efficiency are the tasks of this paper.

The paper is organized as follows. Section 2 describes the architecture of HSPA Packet Scheduling. In Section 3, an improved Packet Scheduling Scheme based on QoS parameter is presented. Simulation results are presented and discussed in Section 4. Finally, Section 5 concludes the paper.

\section{HSPA PACKET SCHEDULING ARCHITECTURE}

As the core of HSPA radio resource management, packet scheduling algorithm plays a crucial role in enhancing the link transmission rate. In completing the resource scheduling of the system, scheduling algorithms regard the channel conditions of user equipment as the main factor to consider and take into account the priority of services, current data to be transmitted and the long-term fairness of the users [10].

In order to better adapt to the rapid changes of channels, HSPA technology transfers the scheduling function unit from RNC to Node B. In the HSPA system, based on Node B as shown in Fig. (1), Node B will directly control the transmission rate and transmission time instead of RNC. In each TTI, UE reports to Node B about the current data to be transmitted in the UE cache and available power by uploading the scheduling information. Thus, direct signaling transmission between Node B and UE can be realized and 5 ms TTI can be supported in TD-HSUPA. When UE requests for downlink scheduling resources, Node B quickly uses scheduling permission to indicate the maximum resource available to UE by scheduling methods. When scheduling permission is granted, Node B can get access to the QoS information provided by $\mathrm{RNC}$ or in the scheduling request of UE $[11,12]$.

This Node B-based fast scheduling greatly shortens the control signaling of scheduling and the response delay of UE so as to control the cell throughput more quickly and accurately, obtain greater the uplink throughput and make full use of the limited bandwidth resource.

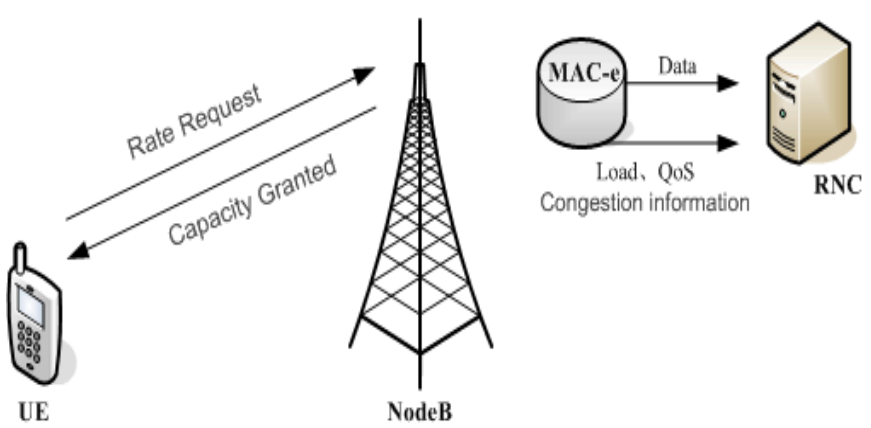

Fig. (1). Node B Scheduling of HSPA.

\section{PROPOSED PACKET SCHEDULING SCHEME BASED ON QOS PARAMETER}

As for PF and M-LWDF are not suitable for hybrid nonreal-time/real-time services, this paper presents a integrated scheduling algorithm based on QoS for mixed services and deals with real-time and non-real-time services by using the three-step time-frequency packet scheduling strategy, so as to meet the requirements of QoS parameters, guaranteed bit rate (GBR) of users under scheduling and time delay as far as possible and at the same time, ensure the sending of data need retransmission.

\subsection{System Model}

As shown in Fig. (2), the system model consists of scheduling inspection, time domain packet scheduling and frequency domain packet scheduling.

The scheduling inspection module identifies the schedulable UE by whether there is HARQ data need retransmission, whether idle process exists and whether there is any data in data buffer (RLC/PDCP) and stores these UEs into the time domain packet scheduling chain table [13, 14].

In the time domain packet scheduling (TDPS) module, the first step is to determine the service type of the user, then identify the time domain packet scheduling (TDPS) priority of the mixed services by the algorithm proposed and select $\mathrm{N}$ $\max$ users with the highest priority. If the number of schedulable users is less than $\mathrm{N}_{\max }$, select all and store the UEs in the frequency domain packet scheduling chain table. Scheduling in this way can be more precise with reduced errors.

Frequency domain packet scheduling (FDPS) module performs frequency domain packet scheduling and resource allocation to the UEs in the frequency domain packet scheduling chain table, distributes VRB serial number to each UE and identifies the MCS used by each UE, quantity of transmission block (Trans- Port Block, TB) and each transport block size (Transport Block Size, TBS).

\subsection{Scheduling Algorithm Based on QoS}

The scheduling discipline based on QoS presented in this paper is different from that of the traditional algorithm. Priority of this algorithm is a comprehensive one which is calculated based on the comprehensive factors, including the queuing status of each user, QoS requirements and the sum of speeds on each channel. This algorithm firstly gives that 
real-time service scheduling is prior to non-real-time one and the priority of real-time service can be expressed as (1):

$Q_{i}(n)=\frac{\sum_{k=1}^{K} r_{i, k}(n)}{R_{i}(n) \times K}+\delta \times \frac{\sum_{j=1}^{N_{i}(n)} \frac{\beta_{i, j} \times L_{i, j}(n)}{D_{i, j}(n)}}{\sum_{k=1}^{K} r_{i, k}(n)}$

In the formula, $\mathrm{K}$ is the number of sub-channels; $\delta$ is adjustable weighting parameter; $\mathrm{Ni}(\mathrm{n})$ is the packet number of TTI user i the buffer queue; $\beta_{i, j}$ is the priority coefficient of the $\mathrm{j}$ packet of user $\mathrm{i}$ in the buffer queue which is determined by QoS requirements of this packet (such as BER, delay jitter); $L_{i, j}(n)$ is packet length; $D_{i, j}(n)$ is the distance from the current delay of $\mathrm{j}$ in TTI packet to its maximum tolerable delay; $r_{i, k}(n)$ is the data transmission speed of user i on carrier $\mathrm{K}$; $\mathrm{R} i(n)$ is the average throughput of user i by time $\mathrm{N}$. The scheduling goal Goal $i(n)$ can be expressed as (2):

$\operatorname{Goal}_{i}(n)=\sum_{j=1}^{N_{i}} \frac{L_{i, j}(n)}{D_{i, i}(n)}$

In this formula, Goal i (n) is the sum of the minimum transmission rates required by the packets in the buffer queue based on their upper limit of time delay. Scheduling goal Goal i (n) reflects the guarantee to the delay requirements of algorithm on real-time service.

The process of scheduling algorithm as follows:

Step 1 When the system is mixed with real-time and nonreal-time services, scheduling real-time service first.

Step 2 In each TTI, prioritize the users of real-time service;

Step 3 The users of real-time services obtain their channel options in accordance with their priority level.

Step 4 Users obtained the channel options can choose the best sub-channel for them from the residual sub-channels to transmit data; the number of the sub-channel is determined by $\operatorname{Goal}_{i}(n)$, if no sub-channel is available, the scheduling is terminated.

Step 5 If there is still residual sub-channels available after user's selecting, conduct scheduling to the non-realtime services in the remaining sub-channels based on the scheduling discipline of PF.

\section{SIMULATION AND PERFORMANCE EVALUATION}

The performance evaluation of the proposed packet scheduling scheme based on the QoS parameters is driven by MATLAB simulation software for High-Speed Uplink Packet Access Network. We compare the performance of proposed packet scheduling scheme with $\mathrm{PF}$ algorithm and M-LWDF algorithm. We evaluate the performance in terms of average packet delay and cell throughput.

When the user regard the mixed services as the main service, do scheduling by using different algorithms and the simulation parameters are as shown in Table $\mathbf{1}$ :

Table 1. Simulation Parameters

\begin{tabular}{|c|c|}
\hline Channel Models & Rayleigh \\
\hline \hline Real-time user & VoIP/Video Stream \\
\hline Rate of real-time user & $512 \mathrm{kpbs} / 64 \mathrm{kpbs}$ \\
\hline Non-real-time user & HTTP $/$ FTP \\
\hline Rate of non-real-time user & $50 \mathrm{kpbs} / 200 \mathrm{kpbs}$ \\
\hline Ch. Bandwidth & $10 \mathrm{MHz}$ \\
\hline Simulation time & $3 \mathrm{~min}$ \\
\hline Packet drop rate & 0.01 \\
\hline TTI & $2 \mathrm{~ms}$ \\
\hline
\end{tabular}

Fig. (3) shows the simulation results for the average throughput of mixed service users under different scheduling algorithms. From the figure, we can see that with the increase of the users, the service load of the system keeps growing so that the average throughput under $\mathrm{PF}$ and $\mathrm{M}$ LWDF algorithms slows down in increase, especially when the user exceeds 30, average throughput under PF algorithm is basically unchanged, whereas in the same condition, the average throughput under improved algorithm will be increased, so that we can draw conclusion that improving the algorithm will present obvious robustness.

As shown in Fig. (4), when the number of user increases gradually, the packet drop rate under $\mathrm{PF}$ algorithm is stabilized at about 0.1 and that of the M-LWDF algorithm is almost around 0.08. But the packet drop rate under improved algorithm is significantly lower than those under the other two algorithms. On one hand, this is because that the great

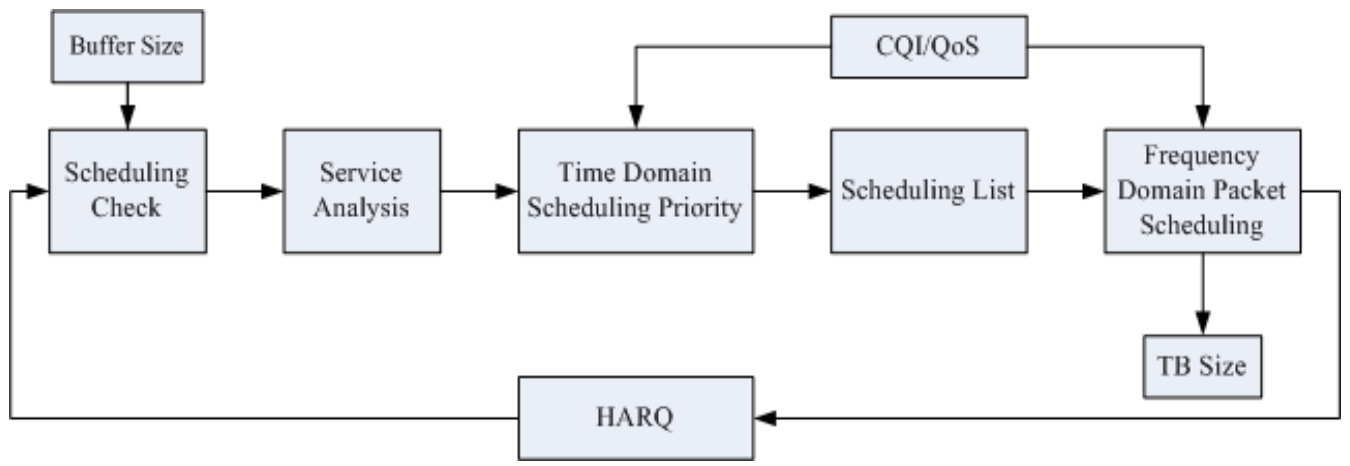

Fig. (2). Three-step time-frequency packet scheduling architecture. 
enhancement in a real-time user throughput will result in the decrease in packet drop rate. On the other hand, the reduced service time for non-real-time service will decrease the throughput which will have an adverse effect on the packet drop rate. Therefore, in general, the average packet drop rate is around 0.055 .

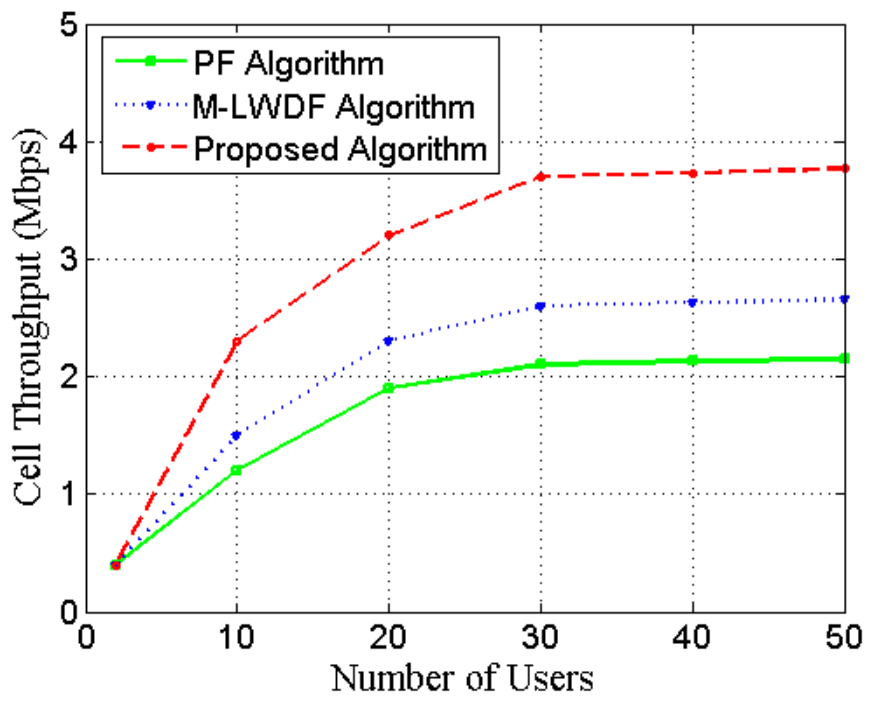

Fig. (3). The average throughput of different scheduling algorithms.

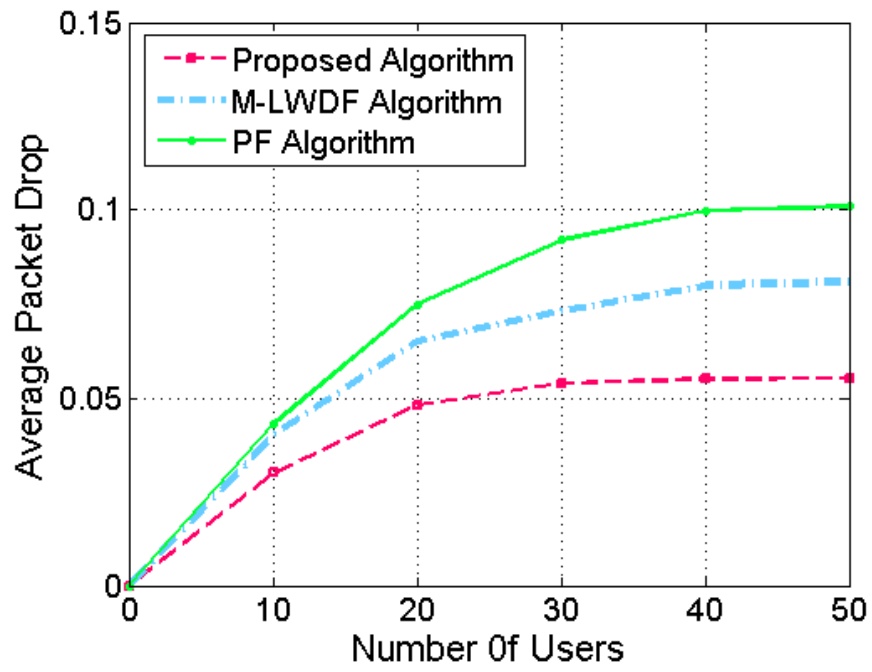

Fig. (4). The packet drop rate of different scheduling algorithms.

\section{CONCLUSION}

In this paper, we have introduced a three step packet scheduling scheme and proposed a packet scheduling algorithm based on QoS parameter for mixed service applications in HSPA systems, in order to meet the actual QoS needs of the different systems. The simulation results show that the performance efficiency of HSPA systems employed proposed algorithm is superior compared to the conventional PF and M-
LWDF algorithm in average packet drop and average cell throughput.

\section{CONFLICT OF INTEREST}

The authors confirm that this article content has no conflict of interest.

\section{ACKNOWLEDGEMENTS}

This work was supported in part by the Research Innovation Program for College Graduates of Jiangsu Province (CXLX12_0238).

\section{REFERENCES}

[1] El-Atty, S. M. Abd, and K. Lizos, "Enhanced uplink scheduling for continuous connectivity in high speed packet access systems", International Journal of Communications, Network and System Sciences, vol. 5, pp. 446-453, 2012.

[2] H. Holma, and A. Toskala, "HSDPA/HSUPA for UMTS high speed radio access for mobile communications" John Wiley \& Sons Ltd., Hoboken, 2006

[3] Y. J. Kang, J. Kim, D. K. Sung, and S. Lee, "Hybrid scheduling algorithm for guaranteeing QoS of Real-Time traffic in high speed uplink packet access (HSUPA)," IEEE 18th International Symposium on Per-sonal, Indoor and Mobile Radio Communications, Athens, 3-7 September 2007, pp. 1-5.

[4] Z. Qian, and Y.-Q. Zhang, "Cross-layer design for QoS support in multihop wireless networks", Proceedings of the IEEE, vol. 96, pp. 64$76,2008$.

[5] L. I. U. Yang, and J. I. Xi sheng, "Cross-layer packet scheduling scheme for supporting QoS of real-time services", Application Research of Computers, vol. 5, pp. 63-68, 2011.

[6] X. Yong-Ju, et al. "Analysis of M-LWDF fairness and an enhanced MLWDF packet scheduling mechanism." The Journal of China Universities of Posts and Telecommunications, vol. 18, pp. 82-88, May 2011.

[7] L. Ju Yong, et al. "Dynamic parameter adaptation for M-LWDF/MLWWF scheduling," Wireless Communications, IEEE Transactions, Vol. 11, pp. 927-937, 2012.

[8] M.R. Heidarinezhad and A. Rajaei, "A QoS-aware packet scheduling scheme in high-speed downlink packet access networks," IEEE Journal of Research, vol. 58, pp. 247-251, 2012.

[9] 3GPP TR 23.107 v 5.13.0, "Technical Specification Group Services and System Aspect; Quality of Service (QoS) Concept and Architecture", Release 5, 2005.

[10] D. C. Dimitrova, J. L. van de n Berg, G. J. Heijenk and R. Litjens, "Flow level performance comparison of packet scheduling schemes for UMTS EUL," Proceedings of 6th International Conference on Wired/Wireless Internet Communications, Tampere, 28-30, pp. 27-40, 2008.

[11] Y. J. Kang, J. Kim, D. K. Sung and S. Lee, "Hybrid scheduling algorithm for guaranteeing QoS of real- time traffic in High Speed Uplink Packet Access (HSUPA)," IEEE 18th International Symposium on Personal, Indoor and Mobile Radio Communications, Athens, vol. 3, pp. 1-5, September, 2007.

[12] 3GPP TS25.433v5.16.0, "UTRAN Iub Interface Node B Application Part (NBAP) Signalling", October 2006.

[13] H. Chiao-Yin, et al. "An intelligent HARQ scheme for HSDPA" Vehicular Technology, IEEE Transactions, vol. 60, pp. 1602-1611, 2011.

[14] Y. Woon-Young, et al. "Power-efficient scheduling for voice services in high-speed packet access systems," IEICE Electronics Express, vol. 8, pp. 76-82, 2011. 